\title{
The inclusion of real world evidence in clinical development planning
}

\author{
Reynaldo Martina ${ }^{1,2^{*}}$ D, David Jenkins ${ }^{1,3}$, Sylwia Bujkiewicz ${ }^{1}$, Pascale Dequen ${ }^{1,4}$, Keith Abrams $^{1}$ and on behalf of \\ GetReal Workpackage 1
}

\begin{abstract}
Background: When designing studies it is common to search the literature to investigate variability estimates to use in sample size calculations. Proprietary data of previously designed trials in a particular indication are also used to obtain estimates of variability. Estimates of treatment effects are typically obtained from randomised controlled clinical trials (RCTs). Based on the observed estimates of treatment effect, variability and the minimum clinical relevant difference to detect, the sample size for a subsequent trial is estimated. However, data from real world evidence (RWE) studies, such as observational studies and other interventional studies in patients in routine clinical practice, are not widely used in a systematic manner when designing studies. In this paper, we propose a framework for inclusion of RWE in planning of a clinical development programme.

Methods: In our proposed approach, all evidence, from both RCTs and RWE (i.e. from studies in routine clinical practice), available at the time of designing of a new clinical trial is combined in a Bayesian network meta-analysis (NMA). The results can be used to inform the design of the next clinical trial in the programme. The NMA was performed at key milestones, such as at the end of the phase II trial and prior to the design of key phase III studies. To illustrate the methods, we designed an alternative clinical development programme in multiple sclerosis using RWE through clinical trial simulations.
\end{abstract}

Results: Inclusion of RWE in the NMA and the resulting trial simulations demonstrated that 284 patients per arm were needed to achieve 90\% power to detect effects of predetermined size in the TRANSFORMS study. For the FREEDOMS and FREEDOMS II clinical trials, 189 patients per arm were required. Overall there was a reduction in sample size of at least $40 \%$ across the three phase III studies, which translated to a time savings of at least 6 months for the undertaking of the fingolimod phase III programme.

Conclusion: The use of RWE resulted in a reduced sample size of the pivotal phase III studies, which led to substantial time savings compared to the approach of sample size calculations without RWE.

Keywords: Network meta-analysis, Relapse rate, Negative binomial model, Clinical development plan, Sample size, Clinical trial simulation

\section{Background}

The drug development process is generally divided into different phases. Trials up to phase II are considered exploratory. Phase III trials are generally designed based on the results of the phase II trials. Some design elements of the phase III trials occasionally also include evidence

\footnotetext{
* Correspondence: reynaldo.martina@liverpool.ac.uk

${ }^{1}$ Department of Health Sciences, University of Leicester, University Road, Leicester, UK

${ }^{2}$ Department of Biostatistics, University of Liverpool, 1-5 Brownlow Street, Liverpool, UK

Full list of author information is available at the end of the article
}

from other relevant randomised controlled trials (RCTs). A recent survey among participants of an International Clinical Trials Methodology Conference confirmed that evidence synthesis is not routinely used in the design and analysis of clinical trials [1]. The use of real world evidence (RWE), such as that from observational and pragmatic studies, in the evaluation of new health technologies and in clinical development programmes has recently become a prominent topic of an international debate [2]. Sutton et al. [3] provided a framework for the synthesis of available evidence with the aim of maximising the use

(C) The Author(s). 2018 Open Access This article is distributed under the terms of the Creative Commons Attribution 4.0 International License (http://creativecommons.org/licenses/by/4.0/), which permits unrestricted use, distribution, and 
of relevant information from existing data sources and thus reducing the need for future studies. Reducing the need for, or at least the size of, future studies has a significant impact on the cost of drug development, and potentially this can be achieved if, for example, the synthesis of available evidence results in more precise estimates of effectiveness. The use of RWE is currently being explored as the way toward bridging the effectiveness-efficacy gap $[4,5]$, i.e. the difference between the efficacy observed in RCTs and the more general effectiveness observed in trials conducted under general practice (real world conditions). To our knowledge, RWE has not been widely included in drug development programmes, for example, to inform future (phase III) studies. However, there have been a few published examples of the use of meta-analysis to inform sample size [6] and the design of future trials [7-9].

The use of only phase II data to design phase III trials has been met with criticism due to the nature of the phase II trials used, which are generally exploratory in nature, and the lack of reproducibility of sample sizes and clinical results of the phase III trials. For example, Tomblyn and Rizzo [10] reported that the use of phase II trial data can be misguided in clinical practice due to the exploratory nature of the trials. Zia et al. [11] compared outcomes of phase II studies with subsequent randomised controlled studies using identical chemotherapeutic regimens. It was reported that $85 \%$ of the phase III studies had lower response rates than those of the preceding phase II trial and the response rates were on average about $12.9 \%$ lower in the phase III studies compared to those of phase II. The lower response rates indicate that these phase III studies were not sufficiently powered. The issues of underpowered studies and poor assumptions in sample size calculations were also reported in the work of Vickers [12] and Charles et al. [13].

De Ridder [14] did not limit the design of a phase III trial to the use of the reported phase II data, but performed modelling and simulation of the phase II data to investigate key design issues such as sample size, the doses or trial duration. DeSantis and Zhu [15] undertook a mixed treatment comparisons meta-analysis and used the results to inform the power and design of a future hypothetical trial. Cameron et al. [16] provided an overview of the challenges and opportunities to include randomised and non-randomised clinical trials in a network meta-analysis for assessing the safety and effectiveness of medical treatments.

The aim of this paper is to describe a novel strategy for inclusion of all available RCT data and RWE in a step-wise approach. By performing network meta-analyses (NMAs) at key stages of development, informed decisions can be made based on all available data. We aim to synthesise the available evidence from phase II and phase III RCTs, including drug trials in real world conditions, and illustrate how the obtained information can be applied to design phase III drug trials in a clinical development programme.

\section{Methods}

The following sections describe the strategy for including RWE in the drug development process in a step-wise and recursive approach. The proposed method is an extension of the approach taken by DeSantis and Zhu [15] and Sutton et al. [6], who claimed that basing the sample size of a new trial on an updated meta-analysis may make more sense than powering the trial in isolation.

We consider the sequential implementation of NMAs that include RCTs and RWE available at key stages of development. The methods are illustrated using an example development for relapsing remitting multiple sclerosis (RRMS).

The annualised relapse rate (ARR) was the primary variable in the development programme and RWE studies obtained. This variable is used throughout this manuscript to illustrate the proposed strategy.

\section{Strategy for the inclusion of RWE in the drug development process}

The procedure performed to include RWE at key stages of development can be summarised in the following steps:

Step 1

(a). Use NMA (to include phase II trial [17] and all RCT and RWE available prior to the design and execution of the TRANSFORMS trial [18]) to obtain estimates of relative effectiveness.

(b). Perform NMAs sequentially in time to include each phase III trial separately in the meta-analysis of all earlier studies, and then finally include all RCTs together.

(c). Extract ARR and standard error from the NMA to use in simulation of alternative development strategies.

Step 2

(a). Use estimates from the NMA based on the predictive distribution [19] to simulate the effects and confidence interval of a future alternative phase III trial (by sampling from a negative binomial distribution).

(b). Assess how many patients will be needed to execute a similar phase III trial with sufficient power (90\%), whilst the probability of falsely claiming effectiveness remains low $(<5 \%)$.

Step 3

Compare the size of the original trial with the size of the simulated trial.

Step 4

(a). Investigate whether the original trial could have been executed successfully with the alternative 
number of patients by analysing the original trial with a lower number of patents.

(b). Using the (lower) number of patients obtained from the simulated trials, run clinical trial simulations assuming the treatment effects are as observed in the reported clinical trials.

(c). Assess power and false positive rate.

Step 5

Include the size and relative effect estimates of simulated trials instead of the original trial results in an NMA to investigate whether or not there is a difference in the totality of evidence of effectiveness between the original NMA and the NMA based on simulated results.

Steps 2 through 4 are repeated for each phase III trial. The following section describes the example development used to illustrate the proposed strategy. The sections titled Network meta-analysis methods and Clinical trial simulations describe the NMA and the clinical trial simulations. The applied software is summarised in the section titled Software.

\section{Illustrative example}

This work has been undertaken as part of the Innovative Medicines Initiative (IMI). As part of this initiative, RCT information was made available by a sponsor to illustrate the methodological proposals described in this manuscript. Hence, the development studies of fingolimod have been used in this illustrative example. The sequential NMA was designed to include RWE available after the execution of the phase II trial of fingolimod [17] and prior to the design and execution of the TRANSFORMS [18], FREEDOMS [20] AND FREEDOMS II [21] phase III RCTs.
The primary variable in the phase III trials was the ARR.

A brief description of the pivotal studies in this illustrative development programme is provided in Table 1 . The table includes the study reference, treatments in each study, number of patients in each study, primary and key secondary outcomes and whether or not the power and/or statistical significance level was included in the publication. The information included in the table was obtained through a literature search.

Table 2 summarises the RWE studies included in the NMA, the treatments, the number of subjects, number of relapses, the person years and the Expanded Disability Status Scale (EDSS). The studies were obtained through a literature search. The ARR is estimated by dividing the number of relapses by the person years.

General guidance regarding how to search for and select studies to be included in an NMA can be found in [22] and will not be further discussed in this manuscript.

\section{Network diagram}

Figure 1 shows the network diagrams including all RCTs (including the phase II trial) and RWE studies separately. Figure 2 shows the integrated network conducted up to the time of health technology assessment (HTA) submissions for fingolimod. There were 23 studies included in the NMA. Fourteen of these studies were RCTs and 9 studies were from RWE sources.

\section{Network-meta analysis methods}

A random effects NMA that allows for variation of treatment effects across studies [23] was undertaken to synthesise data from both sources of evidence: the RCT data and RWE. The NMA was applied to both datasets individually and then to the two sources of evidence combined. In the combined meta-analysis model RWE was included at face

Table 1 Summary of pivotal trials in the illustrative development plan

\begin{tabular}{|c|c|c|c|c|c|}
\hline Study & Treatments & $\begin{array}{l}\text { No. of } \\
\text { patients } \\
(\mathrm{N})\end{array}$ & Primary outcome & Relevant secondary outcomes & Power/significance level \\
\hline Phase II [17] & $\begin{array}{l}\text { Placebo or fingolimod } \\
1.25 \mathrm{mg} \text { or fingolimod } \\
5 \mathrm{mg}\end{array}$ & 281 & $\begin{array}{l}\text { Hamburg Quality of Life } \\
\text { Questionnaire (HAQUAMS) } \\
\text { and Beck Depression } \\
\text { Inventory second edition } \\
\text { (BDI-II) }\end{array}$ & Not published & Not published \\
\hline $\begin{array}{l}\text { TRANSFORMS } \\
{[18]}\end{array}$ & $\begin{array}{l}\text { Interferon beta-1a or } \\
\text { fingolimod } 0.5 \mathrm{mg} \text { or } \\
\text { fingolimod } 1.25 \mathrm{mg}\end{array}$ & 1292 & Annualised relapse rate & $\begin{array}{l}\text { Change in Expanded Disabiity } \\
\text { Status Scale (EDSS) score }\end{array}$ & 90\%/5\% (two-sided) \\
\hline $\begin{array}{l}\text { FREEDOMS } \\
{[20]}\end{array}$ & $\begin{array}{l}\text { Placebo or fingolimod } \\
0.5 \mathrm{mg} \text { or fingolimod } \\
1.25 \mathrm{mg}\end{array}$ & 1272 & Annualised relapse rate & Change in EDSS score & $\begin{array}{l}\text { Power not published/5\% } \\
\text { (two-sided) }\end{array}$ \\
\hline $\begin{array}{l}\text { FREEDOMS } \| \\
\text { [21] }\end{array}$ & $\begin{array}{l}\text { Placebo or fingolimod } \\
0.5 \mathrm{mg} \text { or fingolimod } \\
1.25 \mathrm{mg}\end{array}$ & 1083 & Annualised relapse rate & Change in EDSS score & 90\%/5\% (two-sided) \\
\hline
\end{tabular}


Table 2 RWE studies included in the NMA and main data extracted per treatment

\begin{tabular}{|c|c|c|c|c|c|}
\hline Study & Treatments & No. of subjects $(M)$ & No. of Relapses & Person years & EDSS \\
\hline \multirow[t]{2}{*}{ Lanzillo (2012) [39] } & Natalizumab & 42 & 10 & 42 & 3.5 \\
\hline & Rebif 44 & 42 & 23 & 42 & \\
\hline \multirow[t]{4}{*}{ Limmroth (2007) [40] } & Avonex & 1094 & 1116 & 2188 & 2.62 \\
\hline & Betaferon & 1034 & 1075 & 2068 & \\
\hline & Rebif 22 & 555 & 588 & 1110 & \\
\hline & Rebif 44 & 185 & 233 & 370 & \\
\hline \multirow[t]{5}{*}{ Halpern (2011) [41] } & Natalizumab & 288 & 21 & 72 & \\
\hline & Avonex & 151 & 7 & 38 & \\
\hline & Rebif 22 & 329 & 22 & 82 & \\
\hline & Betaferon & 144 & 11 & 36 & \\
\hline & Glatiramer acetate & 469 & 25 & 117 & \\
\hline \multirow[t]{3}{*}{ Patti (2006) [42] } & Betaferon & 114 & 137 & 570 & 2.2 \\
\hline & Avonex & 37 & 50 & 185 & \\
\hline & Rebif 22 & 17 & 35 & 85 & \\
\hline \multirow[t]{2}{*}{ Río (2005) [43] } & Placebo & 107 & 288 & 356 & 2.73 \\
\hline & Glatiramer acetate & 101 & 204 & 334 & \\
\hline \multirow[t]{4}{*}{ Haas and Firzlaff (2005) [44] } & Avonex & 79 & 109 & 158 & 2.2 \\
\hline & Betaferon & 77 & 123 & 154 & 2.28 \\
\hline & Glatiramer acetate & 79 & 56 & 158 & 1.98 \\
\hline & & 48 & 59 & 96 & 2.36 \\
\hline \multirow[t]{4}{*}{ Khan et al. (2001) [45] } & Placebo & 15 & 23 & 23 & 2.63 \\
\hline & Avonex & 34 & 41 & 51 & 2.71 \\
\hline & Betaferon & 34 & 28 & 51 & 2.6 \\
\hline & Glatiramer acetate & 39 & 29 & 59 & 2.64 \\
\hline \multirow[t]{2}{*}{ Trojano et al. (2003) [46] } & Betaferon & 209 & 136 & 418 & 2.5 \\
\hline & Avonex & 169 & 120 & 338 & 2.4 \\
\hline \multirow[t]{4}{*}{ Carra et al. (2003) [47] } & Avonex & 26 & 14 & 35 & 2.02 \\
\hline & Rebif 44 & 20 & 2 & 27 & 2.08 \\
\hline & Betaferon & 20 & 11 & 27 & 3.31 \\
\hline & Glatiramer acetate & 30 & 8 & 40 & 2.45 \\
\hline
\end{tabular}

value without weighting [24]. No weights or other adjustments were applied to reduce the impact of RWE data. To account for the different sources of evidence, a Bayesian hierarchical model was used [25].

Let $Y_{i k}$ be the number of relapses in treatment $k$ of study $i$. Then assuming a negative binomial (NBin) distribution, we have:

$$
Y_{i k} \sim \operatorname{NBin}\left(\gamma_{i k}, p_{i k}\right)
$$

where $p_{i k}$ is the probability of a relapse in treatment $k$ of study $i$ and $\gamma_{i k}$ is the rate at which events (relapses) occur in arm $k$ for study $i$.

For further details of the NMA models, including sensitivity analyses, the investigation of fixed and random effects models, the prior distributions on model parameters and the uniform prior distribution (UN $[0,2])$ of the between-subject standard deviation, we refer to Jenkins et al. [26].

Based on the RCTs and RWE studies that have been published in RRMS, NMAs were performed at different stages of the development, after phase II of fingolimod [17] (Step 1) and after each pivotal trial in the development programme. A final NMA was also performed to include all data from RCTs and RWE studies in RRMS available prior to the HTA approval of fingolimod. The results of the NMA were used to simulate alternative phase III studies (Step 2) to investigate whether the early and strategic use of RWE could alter clinical decision making and help design more efficient clinical studies, resulting in lower patient numbers required. Figure 3 provides a graphical illustration of how the NMA was 
RCT network

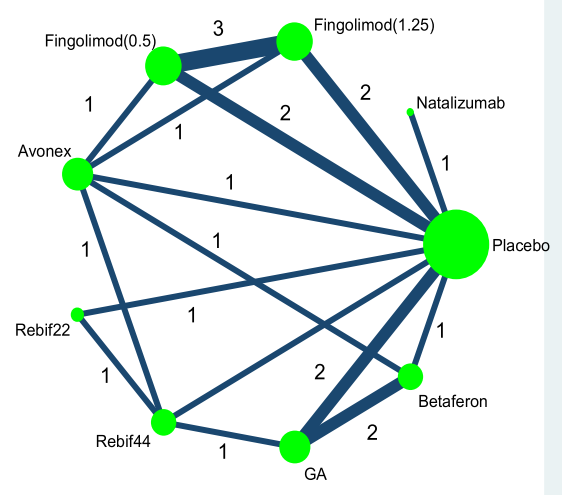

RWE network

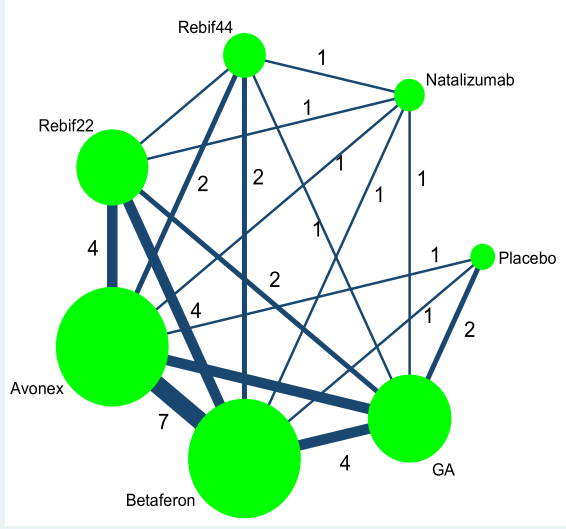

Fig. 1 Left panel: network diagram of RCTs. Right panel: network diagram of RWE studies

used to inform subsequent clinical trial simulations that were performed to illustrate the alternative development strategies.

Starting at the top of Fig. 3, assume there is a network of treatments $\mathrm{A}, \mathrm{B}$ and $\mathrm{C}$, with direct comparisons for $\mathrm{A}$ vs $B$ and $B$ vs $C$ from RCT as well as from RWE data (red lines), and indirect comparisons for treatment A vs treatment B (dotted line in Fig. 3). Following Fig. 3 clockwise, the NMA including RWE can be used to predict relative effectiveness in an RCT. The obtained prediction estimates can be used to simulate alterative trials that compare, say treatment A vs treatment B (green boxes at the bottom of Fig. 3). The simulated effects, variability and duration of the trials can be compared with the results from the original clinical trials. Finally, the NMA can be updated with the simulated RCT results (green arrow in Fig. 3) to investigate the possible impact of future trials on estimates of effect in an NMA. Note that upon completion of the future trial, this can be added to the network instead of the simulated trial to see whether the results of the NMA were as predicted. This process can be repeated as new trials and information become available.

\section{Clinical trial simulations}

The second step in the proposed strategy is to use the estimates from the NMA to simulate the effects and confidence interval of a future alternative phase III trial and assess how many patients will be needed to execute a phase III trial with sufficient power (90\%), whilst the

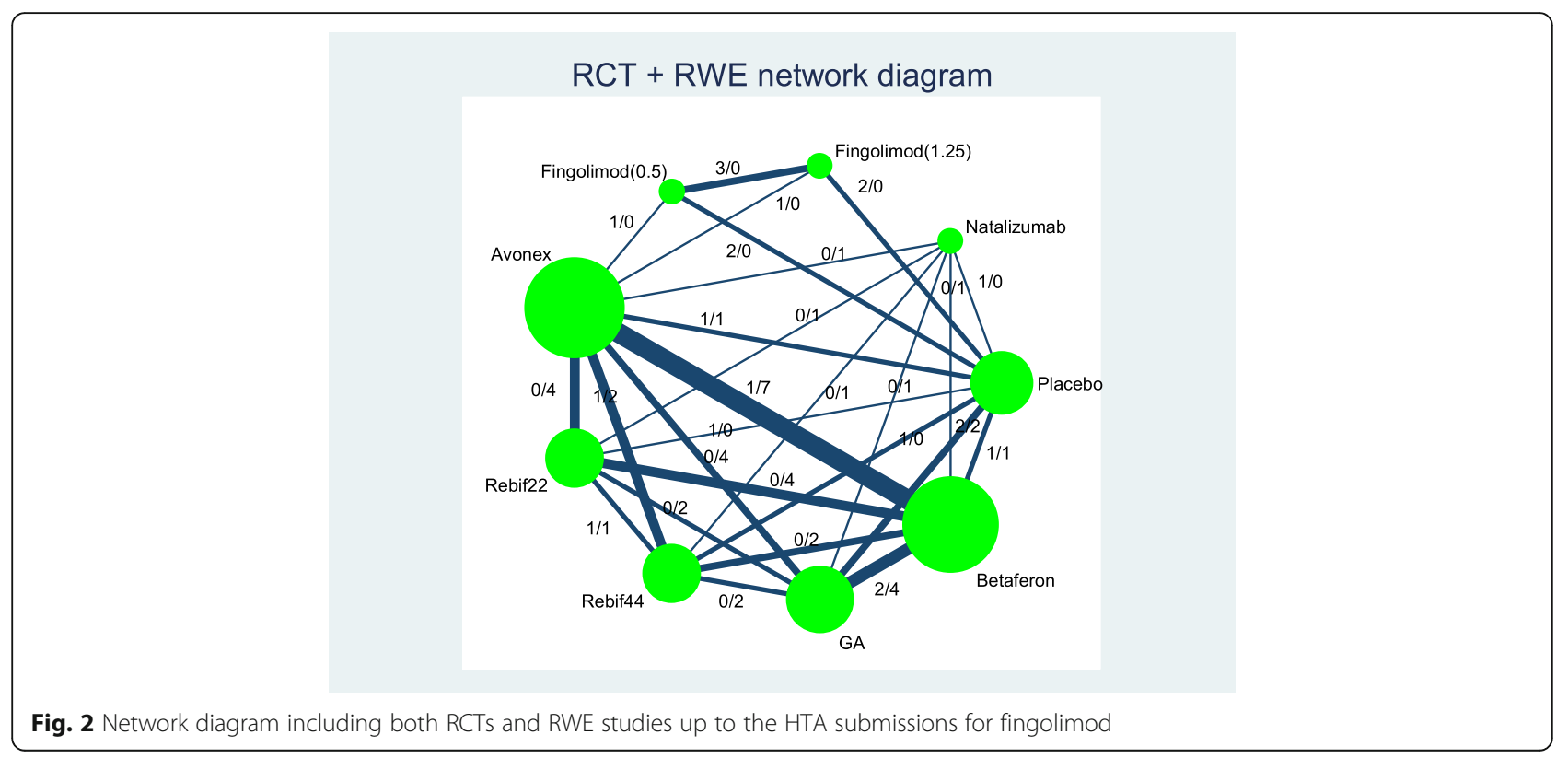




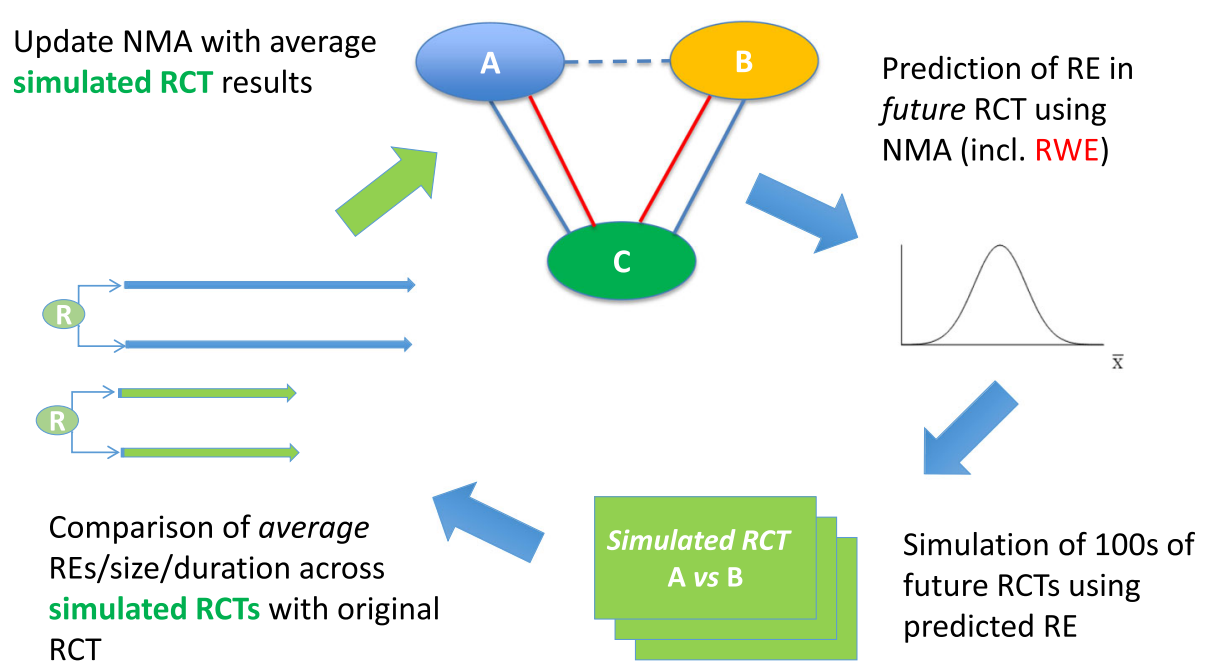

Fig. 3 Graphical illustration of inclusion of RWE in the clinical development strategy

probability of falsely claiming effectiveness remains low $(<5 \%)$.

The estimate of the ARR and corresponding standard error obtained from the NMA which included all trials conducted up to the phase II trial of fingolimod [17], but before the execution of the (next) phase III trial, TRANSFORMS [18], FREEDOMS [20] or FREEDOMS II [21] were used to simulate the next phase III trial of fingolimod.

For a series of sample sizes $\left(n_{1}, n_{2}, \ldots\right), 1000$ random samples were drawn from a negative binomial distribution to represent 1000 clinical trials of size $n_{k}$. So for each trial, $n_{k}$ samples were drawn from the negative binomial distribution $(Y \sim \operatorname{NBin}(r, p))$, where the random variable $Y$ is the number of "failures" (no occurrence of a relapse) before the $r^{\text {th }}$ "success", in this case, the occurrence of a relapse. This procedure was repeated 1000 times.

Following the repeated sampling from the negative binomial distribution, a negative binomial regression was then carried out to compare treatments for each of the 1000 (simulated) clinical trials.

A general negative binomial model is defined by:

$$
\log y_{k}=\beta_{0}+\beta_{1} x_{k 1}+\epsilon_{k}=\mu_{k}+\epsilon_{k}
$$

where $y_{k}$ is a random variable with a negative binomial distribution if we assume that the distribution of $\tau_{k}$ $=e^{\epsilon_{k}}$ has a gamma distribution [27] and $x_{k 1}$ is the indicator variable for treatment arm $k$. The NMA was performed on aggregate data, and as a result, no covariates were included in the negative binomial regression to minimise bias [28]. If individual patient data (IPD) are available, then the covariates known to have an impact on the response parameter can be included.
In Step 3 each trial was then analysed to investigate the number of patients needed to execute that trial, the power of the trial, i.e. Probability(reject the null hypothesis $\mathrm{H}_{0} \mid \mathrm{H}_{1}$ is true) and the probability of falsely claiming effectiveness, i.e. Probability(reject the null hypothesis $\mathrm{H}_{0} \mid \mathrm{H}_{0}$ is true), i.e. the false positive rate of that trial. The trials were analysed using a negative binomial regression model. For set levels of power the numbers of patients required were estimated. Similarly, for varying levels of patients, including the number of patients needed in the original trials, we estimated the number of times (out of 1000) that the null hypothesis was rejected when it is assumed that the "true" state is that treatments were superior to placebo (power) and the number of times (out of 1000) that the null hypothesis was rejected when in fact the "true" state is that the treatments were not superior to placebo (false positive rate).

These results were compared with those from the original trials.

This process can then be repeated at each key stage of development, i.e. before the start of the next (pivotal) phase III trial. Data on relapses were simulated from the results of the NMA. Based on the results of the NMA, patients of an alternative TRANSFORMS trial were simulated and analysed using a negative binomial regression model as described above. Alternative phase III trials were then simulated, and the number of patients needed to execute these simulated trials was evaluated. Subsequently, the published results of the ARR and 95\% confidence intervals were then used to simulate whether or not the TRANSFORMS could have been executed with fewer patients (Step 4). Steps 2, 3 and 4 are repeated after each completed pivotal trial in the programme. In the last step (Step 5), the size and relative effect estimates of simulated trials instead of the original trial results 
were included in an NMA to investigate whether or not there is a difference in the totality of evidence of effectiveness between the original NMA and the NMA based on simulated results.

Following the clinical trial simulations, the sample sizes of the original studies and recruitment time were contrasted to those from the simulated studies. Based on the reported recruitment in the reported studies, the average recruitment rate (number of patients per month) was calculated and applied to the simulated scenarios. The recruitment time of the alternative strategies assumed this recruitment rate, and the time to recruitment was estimated accordingly.

Plots of treatment rankings were produced to compare the rankings of the original synthesised data and the new (simulated) alternative studies to investigate whether the treatment rankings were altered based on the proposed strategy.

\section{Software}

The NMAs were performed in WinBUGS [29]. The clinical trial simulations were performed in the $\mathrm{R}$ programming language [30]. The graphical displays were created using STATA (network diagrams) [31], R (power curves) [30] and Microsoft Projects (for the figure of recruitment time; see Fig. 7 in the subsection titled Results of clinical trial simulations).

\section{Results}

\section{Results of NMA}

Including RWE studies not only increased the evidence base, but also the number of treatment comparisons not considered within the RCTs (see also Figs. 1 and 2). The phase II trial provided additional evidence for the comparison between fingolimod $1.25 \mathrm{mg}$ and placebo.

Table 3 displays the ARR ratios (and standard errors) from an NMA of all RCTs (above the diagonal) and those obtained from the NMA of the RCTs and RWE combined (below the diagonal).

The ARR can be interpreted as the mean number of relapses per year. The ratio is the average relapse rate in the experimental arm compared to the control; e.g. the rate ratio of fingolimod $(1.25 \mathrm{mg})$ over placebo is approximately 0.46 , indicating that a patient on fingolimod has a $54 \%$ lower relapse rate compared to a patient on placebo.

The results of the NMAs that included all trials including the phase II trial of fingolimod were used to investigate the alternative clinical development strategies as described in the following section.

\section{Results of clinical trial simulations}

Figure 4 shows the power curve of the simulated TRANSFORMS trial. The treatment effects used to simulate the power of an alternative TRANSFORMS trial were obtained from the NMA (RCT and RWE) that included the phase II trial. The figure shows that the power of the simulated TRANSFORMS trial exceeded $90 \%$ at 284 patients per arm (568 in total for a two-arm trial). The original TRANSFORMS trial was designed to have $90 \%$ power and recruited approximately 420 patients per arm (840 in total for a two-arm trial). The clinical trial simulations showed that the probability of falsely claiming superiority of the experimental drug vs standard of care was less than $5 \%$. Note that the chosen significance level in the design of the TRANSFORMS trial was $5 \%$ [18].

The reduced sample size implies a potential savings of $30 \%$ of the originally planned sample size if all available evidence (including RWE) is used to design the trial.

Following the clinical trial simulations using RWE in conjunction with the phase II trial of fingolimod to design an alternative TRANSFORMS trial, the published results of the TRANSFORMS trial were analysed assuming that 284 patients per arm were recruited. Clinical trial simulations showed that the probability of observing effects (thus

Table 3 Matrix table of annualised relapse rate ratios (standard errors) for an NMA of all RCTs (above the diagonal) and those obtained from the NMA of the RCTs and RWE combined (below the illustrated diagonal). Results presented at face value with no adjustments made for the inclusion of RWE

\begin{tabular}{|c|c|c|c|c|c|c|c|c|c|}
\hline & Placebo & Nataluzimab & Fingolimod 1.25 & Fingolimod 0.5 & Avonex & Rebif 22 & Rebif 44 & Copaxone & Betaferon \\
\hline Placebo & & $0.314(0.03)$ & $0.462(0.03)$ & $0.423(0.03)$ & $0.832(0.06)$ & $0.727(0.07)$ & $0.679(0.05)$ & $0.659(0.04)$ & $0.670(0.05)$ \\
\hline Nataluzimab & $0.407(0.07)$ & & $1.488(0.20)$ & $1.361(0.20)$ & $2.677(0.30)$ & $2.336(0.30)$ & $2.183(0.30)$ & $2.120(0.30)$ & $2.157(0.3)$ \\
\hline Fingolimod 1.25 & $0.455(0.05)$ & $1.150(0.23)$ & & $0.918(0.08)$ & $1.808(0.17)$ & $1.581(0.19)$ & $1.476(0.15)$ & $1.433(0.14)$ & $1.458(0.15)$ \\
\hline Fingolimod 0.5 & $0.413(0.05)$ & $1.045(0.22)$ & $0.916(0.12)$ & & 1.977 (0.19) & $1.728(0.21)$ & $1.614(0.17$ & $1.567(0.16)$ & $1.594(0.17)$ \\
\hline Avonex & $0.783(0.07)$ & $1.977(0.36)$ & $1.742(0.24)$ & $1.920(0.27)$ & & $0.877(0.09)$ & $0.818(0.06)$ & $0.795(0.07)$ & $0.808(0.07)$ \\
\hline Rebif 22 & $0.766(0.08)$ & $1.933(0.36)$ & $1.706(0.26)$ & $1.880(0.30)$ & $0.982(0.10)$ & & $0.939(0.08)$ & $0.913(0.10)$ & $0.929(0.10)$ \\
\hline Rebif 44 & $0.7482(0.08)$ & $1.887(0.35)$ & $1.666(0.26)$ & $1.837(0.30)$ & $0.959(0.10)$ & $0.983(0.11)$ & & $0.974(0.07)$ & $0.991(0.09$ \\
\hline Copaxone & $0.601(0.05)$ & $1.517(0.28)$ & $1.338(0.19)$ & $1.474(0.21)$ & $0.771(0.07)$ & $0.790(0.09)$ & $0.809(0.09)$ & & $1.019(0.07)$ \\
\hline Betaferon & $0.700(0.07)$ & $1.768(0.32)$ & $1.559(0.22)$ & $1.718(0.25)$ & $0.897(0.07)$ & $0.920(0.09)$ & $0.943(0.1)$ & $1.170(0.11)$ & \\
\hline
\end{tabular}




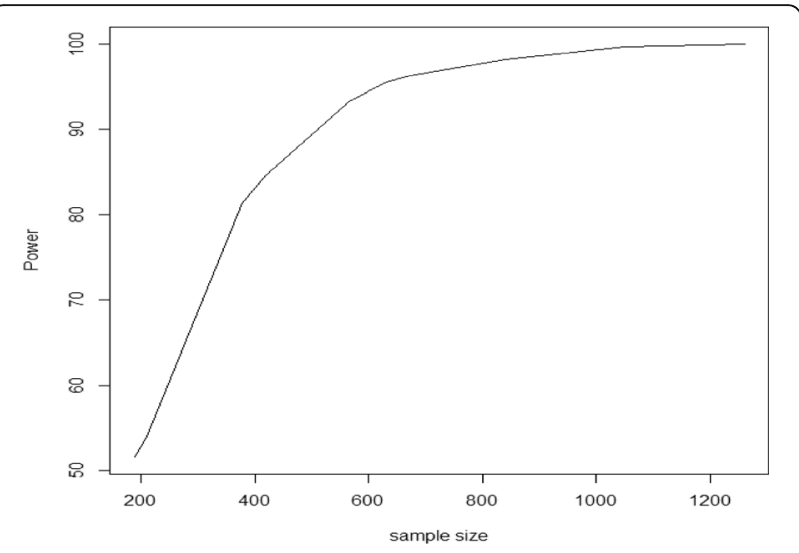

Fig. 4 Power curve of the simulated alternative TRANFORMS study (1000 simulations)

superiority of active over comparator) as reported in the TRANSFORMS trial was $85 \%$. This simulated power was only marginally lower than the prespecified power (90\%) that was used during the design stage of the executed TRANSFORMS trial. However, the $85 \%$ probability of observing a statistically significant difference compared to standard treatment could be achieved with 30\% fewer patients as discussed previously.

Figure 5 outlines the probability of achieving superiority of experimental drug vs standard of care, assuming the results reported in Cohen et al. [18]. The curve illustrates that, assuming the effects reported, 90\% success probability could be achieved with fewer patients, approximately 700 patients in total compared

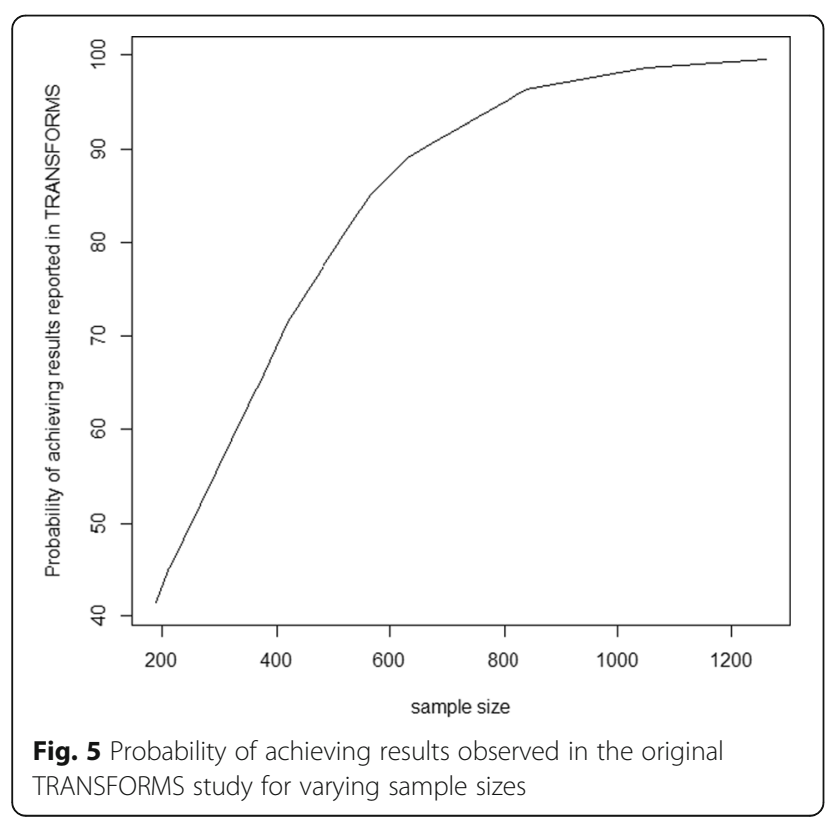

to the 840 patients originally recruited in two arms, a reduction of $17 \%$.

Figure 6 shows the associated power curve for the trial simulations of the simulated TRANSFORMS trial using the effects from the NMA that included RWE prior to TRANSFORMS (black line), the power curve for the TRANSFORMS trial (assuming the published effects are observed, red line) and the power curve using the effects from the NMA that included all available data (green line). This demonstrates that, based on the original design, if RWE were to be included in the development programme, this could result in a significant savings in terms of patient numbers, recruitment time and ultimately costs.

In the alternative strategies, using a predicted trial effect based on an NMA of RCTs and the RWE studies that were available at the time of the design of the TRANSFORMS trial, the power is higher due to the relatively higher effects obtained from all the data in comparison to the increased uncertainty.

Similar evaluations were performed using the FREEDOMS and FREEDOMS II trials. Moreover, it was found that both the FREEDOMS and FREEDOMS II trials could be performed with 189 patients per group if RWE could be considered during the design stage (see alternative III below). This is plausible, since these studies were placebo controlled and not active controlled as the TRANFORMS trial, so fewer patients would be required.

Figure 7 shows the recruitment time of the original TRANSFORMS, FREEDOMS and FREEDOMS II trials and two alternatives based on alternative development strategies.

The following alternative strategies can be considered:

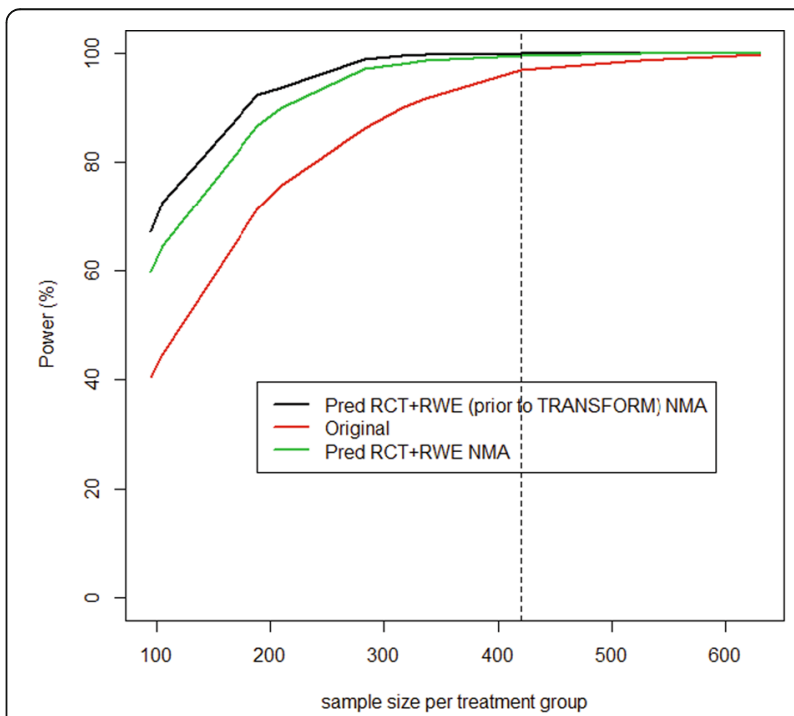

Fig. 6 Power curves based on 1000 trial simulations of a trial of active vs comparator 


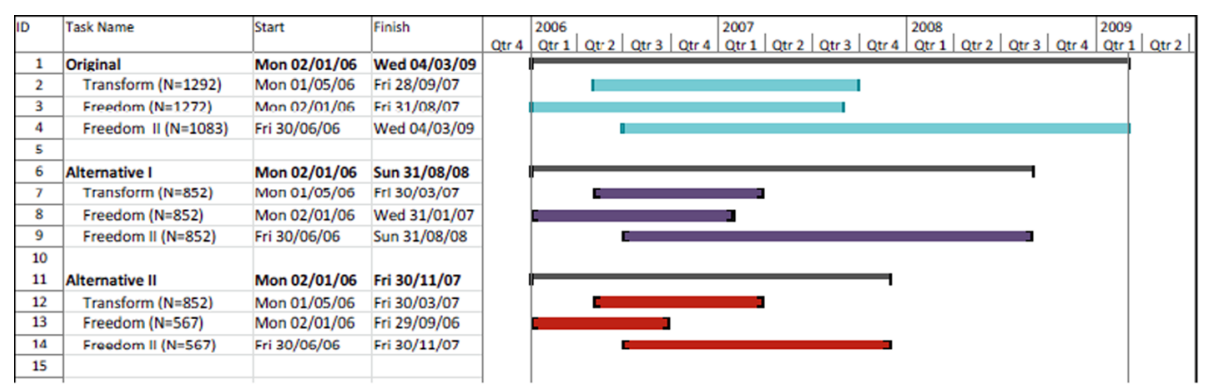

Fig. 7 Recruitment times in original TRANSFORMS and projected recruitment times in simulated TRANSFORMS for two alternatives

Option I (corresponding to alternative I Fig. 7). This option illustrates the original strategy to execute the studies with a similar number of patients. Using RWE to design the TRANSFORMS, the subsequent studies were assumed to require a similar number of patients. The timelines show that using RWE to design the clinical programme could result in a savings of 6 or 7 months on each trial.

Option II (corresponding to alternative II in Fig. 7). Clinical trial simulations of the FREEDOMS trials showed that the FREEDOMS II trial required a similar number of patients as the FREEDOMS trial (567 in total). In this option, the resulting savings would be remarkable: 6 months for TRANSFORMS, 11 months for FREEDOMS and 16 months for FREEDOMS II. Option III (combining alternatives I and II). Based on the number of patients needed to execute the alternative FREEDOMS II trial (mainly a North American population), the FREEDOMS II trial could be executed as a cohort of the FREEDOMS trial. This implies that the FREEDOMS II trial would not be required. The single trial would have more than sufficient power to analyse the patients recruited in FREEDOMS and the North American cohort consisting of 189 patients per arm (567 in total). The combined FREEDOMS trials could require a total of 1134 patients $(2 \times 567)$, and the FREEDOMS II trial would not be required as a separate trial, whilst the alternative FREEDOMS trial would still be marginally smaller than the reported trial with 1272 patients randomised.

Table 4 shows the results from the NMA which included the results of the simulated TRANSFORMS, FREEDOMS and FREEDOMS II trials. Note that the original TRANSFORMS, FREEDOMS and FREEDOMS II results were replaced with the simulated results in this NMA.

The ARR and SE clearly show that the results of the NMA that included the simulated studies were very similar to those from the NMA that included the original trials, with only marginal increases in uncertainty despite the comparatively larger reduction in sample size (see also Table 3). The NMA based on the simulated trials did not alter the treatment rankings seen in previous analyses (See Fig. 8), indicating that the (simulated) smaller studies would not alter the overall evidence of effect. The lower ranked treatments in dark blue (starting from placebo $(\mathrm{P})$ ) from the NMA that included the original trials remained low in rank (dark blue) in the NMA that included the simulated trials. Similarly, the higher ranked treatments (light

Table 4 Matrix table of annualised relapse rate ratios (standard errors) for an NMA of all RCTs, including the simulated TRANFORMS and FREEDOMS studies (above the diagonal) and those obtained from the NMA of the simulated RCTs and RWE combined (below the diagonal). Results presented at face value with no adjustments made for the inclusion of RWE

\begin{tabular}{llllllllll}
\hline & Placebo & Nataluzimab & Fingolimod 1.25 & Fingolimod 0.5 & Avonex & Rebif 22 & Rebif 44 & Copaxone & Betaferon \\
\hline Placebo & & $0.319(0.05)$ & $0.519(0.05)$ & $0.465(0.05)$ & $0.916(0.09)$ & $0.783(0.10)$ & $0.706(0.07)$ & $0.667(0.06)$ & $0.686(0.07)$ \\
Nataluzimab & $0.420(0.08)$ & & $1.666(0.31)$ & $1.492(0.28)$ & $2.940(0.54)$ & $2.370(0.54)$ & $2.265(0.42)$ & $2.140(0.39)$ & $2.157(0.41)$ \\
Fingolimod 1.25 & $0.497(0.07)$ & $1.210(0.28)$ & & $0.901(0.09)$ & $1.781(0.22)$ & $1.437(0.25)$ & $1.373(0.20)$ & $1.297(0.18)$ & $1.335(0.19)$ \\
Fingolimod 0.5 & $0.448(0.07)$ & $1.103(0.26)$ & $0.913(0.13)$ & & $1.989(0.25)$ & $1.606(0.28)$ & $1.534(0.22)$ & $1.450(0.20)$ & $1.491(0.22)$ \\
Avonex & $0.831(0.08)$ & $2.039(0.40)$ & $1.703(0.27)$ & $1.886(0.30)$ & & $0.811(0.13)$ & $0.774(0.09)$ & $0.732(0.09)$ & $0.753(0.09)$ \\
Rebif 22 & $0.798(0.10)$ & $1.957(0.40)$ & $1.639(0.30)$ & $1.814(0.33)$ & $0.965(0.11)$ & & $0.920(0.14)$ & $0.920(0.14)$ & $0.945(0.16)$ \\
Rebif 44 & $0.781(0.10)$ & $1.914(0.38)$ & $1.604(0.29)$ & $1.776(0.32)$ & $0.944(0.11)$ & $0.987(0.13)$ & & $0.951(0.10)$ & $0.980(0.13)$ \\
Copaxone & $0.614(0.06)$ & $1.507(0.30)$ & $1.261(0.21)$ & $1.396(0.23)$ & $0.743(0.08)$ & $0.777(0.10)$ & $0.794(0.10)$ & & $1.033(0.10)$ \\
Betaferon & $0.728(0.08)$ & $1.788(0.35)$ & $1.496(0.25)$ & $1.656(0.28)$ & $0.881(0.08)$ & $0.921(0.10)$ & $0.941(0.11)$ & $1.193(0.12)$ & \\
\hline
\end{tabular}




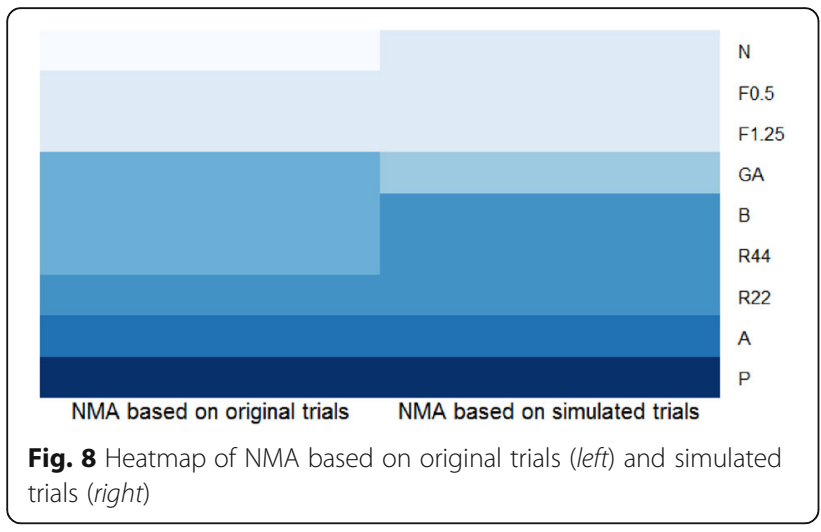

blue) remained higher in rank in both NMAs. The colour code of the intermediate treatments also remained similar in both analyses, indicating no change in their rankings between both NMAs.

Based on these results, one can conclude that in this example the use of RWE at key stages of development could result in smaller clinical trials, limiting the exposure of patients to an inferior treatment, without loss of evidence of effectiveness (see also Table 2). This strategy may result in an earlier regulatory and HTA approval and can contribute to addressing the medical needs of patients and patient groups.

\section{Discussion}

The results from NMAs were used to inform the design of clinical trials in an example clinical development programme. Power curves were created through clinical trial simulations, assuming different scenarios of including RWE in the NMA. There were a number of advantages associated with this approach. We have demonstrated that the efficiency of clinical trial design can be improved (expressed in terms of lower patient numbers in a development plan of studies) by using RCT and RWE data together. We have also shown that inclusion of RWE in the clinical development strategy planning could result in a more efficient clinical development plan compared to having the development strategy be based on a single RCT. Note that the sample size calculations for the phase III studies were based on a Wilcoxon rank sum test, a non-parametric method, whilst our sample size estimations were based on clinical trial simulations using a negative binomial distribution, which is the underlying assumption in the method used for analysing the clinical trial data $[18,20,21]$. As a result, it is unclear what proportion of gain is due to the inclusion of RWE or the fact that the original sample size was based on a Wilcoxon rank sum test. The Wilcoxon rank sum test might have been more conservative in estimating the sample size, leading to the inclusion of more patients, compared to a sample size based on a negative binomial assumption which would correspond to the method used to analyse the data. In general, if distributional assumptions are met, a parametric approach for sample size estimation and data analysis is more powerful than a non-parametric approach [32, 33].

Although we have shown a positive effect (smaller phase III studies) of including RWE data in the development strategy, note that there may be instances when the inclusion of RWE may result in a larger trial in the programme due to increased heterogeneity between studies [8]. This does not necessarily imply that RWE should not have been used, but it may reflect the possibility that the effects observed in an RCT that did not include RWE are not representative of a target population under consideration and a larger trial incorporating knowledge from RWE patients may be more informative.

The RCT data are taken at face value without weighting to illustrate the use of an NMA for clinical trial design purposes. However, more elaborate analyses could be undertaken to weight the RCT and RWE data for rigour, bias and relevance [34]. Moreover, the analyses performed did not account for covariates, because only aggregate RCT and RWE data were available. In the presence of individual patient data, these analyses could be performed accounting for patient baseline characteristics [35]. The use of RWE for sample size calculation is an extension to the use of pairwise meta-analysis to design future trials [6-9]. Fewer patients per study may be needed and fewer pivotal studies required due to the totality of evidence included in the development strategy. As a result, an informed decision regarding effectiveness can be taken earlier, resulting in reduction of cost of clinical development, fewer patients being exposed and ultimately earlier accessibility to effective drugs or alternatively, when the evidence shows lack of effect, early termination of a drug that is not shown to be effective based on the totality of evidence included in the decision-making process. RWE can also provide evidence of effectiveness in the real world not measured in an RCT [36]. The NMA is recommended to be performed at key milestones, such as at the end of the phase II trial and prior to the design of key phase III studies, and repeated when new evidence becomes available. In short, it should be an integral part of the clinical development programme process. We adopted a Bayesian approach to undertaking the NMAs, as this naturally allows predications to be made which are crucial input to the trial simulation process [19]. Alternative approaches may also be considered in conjunction with the proposed strategy to include RWE, such as interim analyses that may include sample size re-estimation approaches, choice of alternative comparators or more flexible (adaptive) clinical trial designs. However, some of the pitfalls of these approaches may include increased sample size [37]. Kairalla et al. [38] described cases where appropriately designed 
adaptive trials could lead to reduced sample size and an increased chance of answering the clinical questions of interest. Kairalla et al. [38] also highlighted areas, such as comparative effectiveness studies, that may benefit from an adaptive design approach. Whatever approach is considered, care should be given to maximise the use of all available evidence and optimise the use of the patient data collected. The proposed strategy could increase the efficiency of flexible designs even further.

\section{Conclusion}

The use of RWE resulted in a reduced sample size of the pivotal phase III studies, which lead to substantial time savings compared to the approach of sample size calculations without RWE. However, further case studies and simulation studies are required to assess the situations when such an approach may be particularly attractive.

\section{Abbreviations}

ARR: Annualised relapse rate; EDSS: Expanded Disability Status Scale; HTA: Health technology assessment; NBIN: Negative binomial; NMA: Network meta-analysis; RCT: Randomised controlled clinical trial; RRMS: Relapsing remitting multiple sclerosis; RWD: Real world data; RWE: Real world evidence

\section{Acknowledgements}

The research leading to these results was conducted as part of the GetReal consortium. For further information refer to www.imi-getreal.eu. This paper only reflects the personal views of the stated authors. The work leading to these results has received support from the Innovative Medicines Initiative Joint Undertaking under grant agreement no. 115546, resources of which are composed of financial contribution from the European Union's Seventh Framework Programme (FP7/2007-2013) and European Federation of Pharmaceutical Industries and Associations (EFPIA) companies' in-kind contribution. KA was also partially supported as a National Institute for Health Research (NIHR) Senior Investigator (NI-SI-0512-10159). SB was also supported by the Medical Research Council (MRC) Methodology Research Programme (New Investigator Research Grant MR/L009854/1).

\section{Funding}

The work leading to these results has received support from the Innovative Medicines Initiative (see also Acknowledgements).

\section{Availability of data and materials}

All data described are publicly available and appropriately referenced in this manuscript. Simulated datasets are available upon request.

\section{Authors' contributions \\ RM, DJ, SB, PD and KA generated the ideas that led to the analyses presented in this manuscript. DJ performed the network meta-analyses; KA and RM performed the clinical trial simulations. SB and PD provided critical review of the analyses and review of the manuscript. All authors read and approved the final manuscript}

\section{Ethics approval and consent to participate}

Not applicable.

\section{Consent for publication}

Not applicable.

\section{Competing interests}

The authors declare that they have no competing interests.

\section{Publisher's Note}

Springer Nature remains neutral with regard to jurisdictional claims in published maps and institutional affiliations.

\section{Author details}

'Department of Health Sciences, University of Leicester, University Road, Leicester, UK. ²Department of Biostatistics, University of Liverpool, 1-5 Brownlow Street, Liverpool, UK. ${ }^{3}$ School of Health Sciences, University of Manchester, Oxford Road, Manchester, UK. ${ }^{4}$ Evidence Synthesis/Health Economics, Visible Analytics Ltd., Union Way, Oxon, UK.

Received: 21 December 2017 Accepted: 28 June 2018

Published online: 29 August 2018

\section{References}

1. Clayton GL, Smith IL, Higgins JP, Mihaylova B, Thorpe B, Cicero R, Lokuge K, Forman JR, Tierney JF, White IR, Sharples LD, Jones HE. The INVEST project: investigating the use of evidence synthesis in the design and analysis of clinical trials. Trials. 2017;18:219-29.

2. Annemans $L$, Aristides M, Kubin M. Real-life data: a growing need. ISPOR connections. 2015;13(5):8-12.

3. Sutton AJ, Cooper NJ, Jones DR. Evidence synthesis as the key to more coherent and efficient research. BMC Med Res Methodol. 2009;9:29.

4. Nordon C, Karcher $\mathrm{H}$, Groenwold RHH, Ankarfeldt MZ, Pichler F, Chevrou-Severac H, Rossignol M, Abbe A, Abenheim L. The efficacyeffectiveness gap: historical background and current conceptualization. Value Health. 2016;19(1):75-81.

5. Ankarfeldt MZ, Adalsteinsson E, Groenwold RHH, Ali MS, Klungel OH. A systematic literature review on the efficacy-effectiveness gap: comparison of randomized controlled trials and observational studies of glucose lowering drugs. Clin Epidemiol. 2017;9:41-51.

6. Sutton AJ, Cooper NJ, Jones DR, Lambert PC, Thompson JR, Abrams KR. Evidence-based sample size calculations based upon updated meta-analysis. Stat Med. 2007;26(12):2479-500.

7. Burke DL, Billingham $\sqcup$, Girling AJ, Riley RD. Meta-analysis of randomized phase II trials to inform subsequent phase III decisions. Trials. 2014;15:346.

8. Nikolakopoulou A, Mavridis D, Salanti G. Using conditional power of network meta-analysis (NMA) to inform the design of future clinical trials. Biom J 00. 2014;1:18

9. Roloff $V$, Higgins JPT, Sutton AJ. Planning future studies based on the conditional power of a meta-analysis. Stat Med. 2013;32:11-24.

10. Tomblyn MR, Rizzo JD. Are there circumstances in which phase 2 study results should be practice-changing? Hematology. 2007;2007:489-92.

11. Zia MI, Siu LL, Pond GR, Chen EX. Comparison of outcomes of phase ॥ studies and subsequent randomized controlled studies using identical chemotherapeutic regimens. J Clin Oncol. 2005;23(28):6982-91.

12. Vickers AJ. Underpowering in randomized trials reporting sample size calculation. J Clin Epidemiol. 2013;56:717-20.

13. Charles P, Giraudeau B, Dechartres A, Baron G, Ravaud P. Reporting of sample size calculation in randomised controlled trials: review. BMJ (Clinical Research Ed.). 2009;338:b1732.

14. De Ridder F. Predicting the outcome of phase III trials using phase II data: a case study of clinical trial simulation in late stage drug development. Basic Clin Pharmacol Toxicol. 2005:96(3):235-41.

15. DeSantis SM, Zhu H. A Bayesian treatment comparisons meta-analysis of treatment for alcohol dependence and implications for planning future trials. Med Decis Mak. 2014;34(7):899-910.

16. Cameron C, Fireman B, Hutton B, Clifford T, Coyle D, Wells G, Dormuth CR, Platt R, Toh S. Network meta-analysis incorporating randomized controlled trials and non-randomized comparative cohort studies for assessing the safety and effectiveness of medical treatments: challenges and opportunities. Syst Rev. 2015;4:147.

17. Montalban X, O'Connor P, Gold SM, de Vera A, Eckert B, Kappos L. Oral fingolimod (FTY720) in relapsing multiple sclerosis: impact on health-related quality of life in a phase II study. Mult Scler. 2011;17(11):1341-50.

18. Cohen JA, Barkhof F, Comi G, Hartung HP, Khatri BO, Montalban X, Pelletier J, Capra R, Gallo P, Izquierdo G, Tiel-Wilck K, de Vera A, Jin J, Stites T, Wu S, Aradhye S, Kappos L, TRANSFORMS Study Group. Oral fingolimod or intramuscular interferon for relapsing multiple sclerosis. N Engl J Med. 2010;362(5):402-15.

19. Spiegelhalter DJ, Abrams KR, Myles JP. Bayesian approaches to clinical trials and health care evaluation. Hoboken: Wiley; 2004.

20. Kappos L, Radue EW, O'Connor P, Polman C, Hohlfeld R, Calabresi P, Selmaj K, Agoropoulou C, Leyk M, Zhang-Auberson L, Burtin P, FREEDOMS Study 
Group. A placebo-controlled trial of oral fingolimod in relapsing multiple sclerosis. N Engl J Med. 2010;362(5):387-401.

21. Calabresi PA, Radue EW, Goodin D, Jeffrey D, Rammohan KW, Reder AT, Vollmer T, Agius MA, Kappos L, Stites T, Li B, Cappiello L, von Rosenstiel P, Lublin FD. Safety and efficacy of fingolimod in patients with relapsingremitting multiple sclerosis (FREEDOMS II): a double-blind, randomised, placebo-controlled, phase 3 trial. Lancet. 2014;13:545-56.

22. Laws A, Kendall R, Hawkins N. A comparison of national guidelines for network meta-analysis. Value Health. 2014;17:642-54.

23. LU G, Ades AE. Combination of direct and indirect evidence in mixed treatment comparisons. Stat Med. 2004;23:3105-24.

24. Ades $\mathrm{AE}$, Welton $\mathrm{N}$, Lu G. Introduction to mixed treatment comparisons. Bristol: University of Bristol. Available from www.bristol.ac.uk. Accessed 11 July 2018.

25. Prevost TC, Abrams KR, Jones DR. Hierarchical models in generalized synthesis of evidence: an example based on studies of breast cancer screening. Stat Med. 2000;19(24):3359-76.

26. Jenkins D, Bujkiewicz S, Martina R, Dequen P, Abrams K. Methods for the inclusion of real world evidence in network meta-analysis. Available from https://arxiv.org/abs/1805.06839. Accessed 18 May 2018.

27. Agresti A. Categorical data analysis ( $3^{\text {rd }}$ edn). Wiley Series in Probability and Statistics 2013.

28. Debray TPA, Moons KGM, van Valkenhoef G, Efthimiou O, Hummel N, Groenwold RHH, Reitsma JB. Get real in individual participant data (IPD) meta-analysis: a review of the methodology. Res Synth Methods. 2015;6(4):293-309.

29. Lunn DJ, Thomas A, Best N, Spiegelhalter D. WinBUGS - a Bayesian modelling framework: concepts, structure, and extensibility. Stat Comput. 2000;10(4):325-37.

30. R core team. R: a language and environment for statistical computing Vienna: The R Foundation for Statistical Computing; 2014.

31. StataCorp. Stata statistical software: release 14. College Station: StataCorp LP; 2015

32. Altman DG, Bland M. Parametric v non-parametric methods for data analysis. BMJ. 2009;338:a3167.

33. Martina R, Kay R, van Maanen R, Ridder A. The analysis of incontinence episodes and other count data in patients with overactive bladder by Poisson and negative binomial regression. Pharm Stat. 2015;14:151-60.

34. Turner RM, Spiegelhalter DJ, Smith GCS, Thompson SG. Bias modelling in evidence synthesis. J R Stat Soc Ser A. 2009;172(1):21-47.

35. Woods B, Hawkins N, Mealing S, Sutton A, Abram WT, Beshai JF, Klein H, Sculphur M, Plummer CJ, Cowie MR. Individual patient data network meta-analysis of mortality effects of implantable cardiac devices. Heart. 2015;101:1800-6.

36. De Lusignan S, Crawford L, Munro N. Creating and using real-world evidence to answer questions about clinical effectiveness. J Innov Health Inform. 2015;22(3):368-73.

37. Gould L. Sample size re-estimation: recent developments and practical considerations. Stat Med. 2001;20(17-18):2625-43.

38. Kairalla JA, Coffey CS, Thomann MA, Miller EK. Adaptive trial designs: a review of barriers and opportunities. Trials. 2012;13:145.

39. Lanzillo R, Quarantelli M, Bonavita S, Ventrella G, Lus G, Vacca G, Prinster A, Orefice G, Tedeschi G, Brescia Morra V. Natalizumab vs interferon beta 1a in relapsing-remitting multiple sclerosis: a head-to-head retrospective study. Acta Neurol Scand. 2012;126(5):306-14.

40. Limmroth V, Malessa R, Zettl UK, Koehler J, Japp G, Haller P. Elias W, Obhof W, Viehöver A, Meier U. Quality assessment in multiple sclerosis therapy (Quasims). J Neurol. 2007;254(1):67-77.

41. Halpern R, Agarwal S, Borton L, Oneacre K, Lopez-Bresnahan MV Adherence and persistence among multiple sclerosis patients after one immunomodulatory therapy failure: retrospective claims analysis. Adv Ther. 2011:28(9):761-75.

42. Patti F, Pappalardo A, Florio C, Politi G, Fiorilla T, Reggio E, Reggio A. Effects of interferon beta-1a and -1b over time: 6-year results of an observational head-to-head study. Acta Neurol Scand. 2006;113(4):241-7.

43. Río J, Rovira A, Tintore M, Sastre-Garriga J, Castillo J, Auger C, Nos C, Comabella M, Tur C, Vidal A, Montalban X. Evaluating the response to glatiramer acetate in relapsing-remitting multiple sclerosis (RRMS) patients. Mult Scler. 2014;20(12):1602-8.

44. Haas J, Firzlaff M. Twenty-four-month comparison of immunomodulatory treatments a retrospective open label study in 308
RRMS patients treated with beta interferons or glatiramer acetate (copaxone). Eur J Neurol. 2005;12(6):425-31.

45. Khan O, Tselis C, Kamholz J, Garbern J, Lewis R, Lisak R. A prospective, open-label treatment trial to compare the effect of IFNb-1a (Avonex), IFNb-1b (Betaseron), and glatiramer acetate (Copaxone) on the relapse rate in relapsing-remitting multiple sclerosis: results after 18 months of therapy. Mult Scler. 2001;7(6):349-53.

46. Trojano M, Liguori M, Paolicelli M, Bosco Zimatore G, De Robertis F, Avolio C, Giuliani F, Fuiani A, Livrea P. Interferon beta in relapsing/remitting multiple sclerosis: an independent postmarketing study in southern Italy. Mult Scler. 2003:9(5):451-7.

47. Carra A, Onaha P, Sinay V, Alvarez F, Luetic G, Bettinelli R, San Pedro E, Rodriguez $\mathrm{L}$. A retrospective, observational study comparing the four available immunomodulatory treatments for relapsing-remitting multiple sclerosis. Eur J Neurol. 2003:10(6):671-6.
Ready to submit your research? Choose BMC and benefit from:

- fast, convenient online submission

- thorough peer review by experienced researchers in your field

- rapid publication on acceptance

- support for research data, including large and complex data types

- gold Open Access which fosters wider collaboration and increased citations

- maximum visibility for your research: over $100 \mathrm{M}$ website views per year

At $\mathrm{BMC}$, research is always in progress.

Learn more biomedcentral.com/submissions 